\title{
Integrated Weed Management in Chilli (Capsicum Annum L.)
}

\author{
D. Krishnamurthy*, B.K. Desai and Maruthi \\ Department of Agronomy, University of Agricultural Sciences, Raichur- 584 104, Karnataka, \\ India \\ *Corresponding author
}

\begin{tabular}{l} 
Keyw or d s \\
$\begin{array}{l}\text { Pendimethalin, } \\
\text { Propaquizapop, } \\
\text { Intercultivation, } \\
\text { density of weeds, } \\
\text { dry weight, chilli }\end{array}$ \\
\hline Article Info \\
$\begin{array}{l}\text { Accepted: } \\
\text { 15 December } 2019 \\
\text { Available Online: } \\
\text { 20 January } 2020\end{array}$ \\
\hline
\end{tabular}

A B S T R A C T

A field experiment was conducted at Main Agricultural Research Station Farm, University of Agricultural Sciences, Raichur in Hyderabad Karnataka region, to study integrated weed management in chilli (Capsicum annum L.). An experiment comprising ten treatments viz. Propaquizafop 10\% EC PoE (postemergence) @ $500 \mathrm{ml} \mathrm{ha}^{-1}$ (50 g a.i.), Propaquizafop 10\% EC PoE@ $625 \mathrm{ml} \mathrm{ha}^{-1}$ (62.5 g a.i.), Propaquizafop 10\% EC PoE @ $750 \mathrm{ml} \mathrm{ha}^{-1}(75 \mathrm{~g}$ a.i.), Propaquizafop 10\% EC PoE @ $1250 \mathrm{ml} \mathrm{ha}^{-1}$ (125 g a.i.), Pendimethalin 30\% EC PRE (preemergence) @ $4160 \mathrm{ml} \mathrm{ha}^{-1}$ (1250 g a.i.), Pendimethalin 30\% EC PRE @ $4160 \mathrm{ml} \mathrm{ha}^{-1}$ (1250 g a.i.) -1 IC at 30 DAT, Propaquizafop 10\% EC PoE @ $625 \mathrm{ml} \mathrm{ha}^{-1}(62.5 \mathrm{~g}$ a.i.) fb (followed by) 1 intercultivation at 30 DAT, Pendimethalin 30\% EC PRE @ $4160 \mathrm{ml} \mathrm{ha}^{-1}$ (1250 g a.i.) fb $10 \%$ EC PoE @ $625 \mathrm{ml} \mathrm{ha}^{-1}$ (62.5 g a.i.) at 2-7 leaf stage, weedy check and control (Hand weeding). All the weed control treatments resulted significant reduction in weed growth in terms of density and dry weight compared to weedy check. Hand weeding thrice at 35, 50 and 65 days after transplanting (DAT) recorded lowest density and dry weight of weeds $\left(12.52 \mathrm{~m}^{-2}\right.$ and $19.99 \mathrm{~g} \mathrm{~m}^{-2}$, respectively) and higher weed control efficiency (WCE) at 45 days after spraying $(88.17 \%)$. The application of pendimethalin $30 \%$ EC PRE @ $4160 \mathrm{ml} \mathrm{ha}^{-1}$ (1250 g a.i.) propaquizafop 10\% EC PoE @ $625 \mathrm{ml} \mathrm{ha}^{-1}$ and propaquizafop 10\% EC PoE @ $1250 \mathrm{ml} \mathrm{ha}^{-1}$ has not recorded any phyto-toxicity on chilli and succeeding fodder cowpea. The maximum green chilli yield $\left(23.2 \mathrm{t} \mathrm{ha}^{-1}\right)$ was recorded three hand weedings at 35, 50 and 65 DAT and lowest green chilli yield was recorded with weedy check $\left(13.5 \mathrm{t} \mathrm{ha}^{-1}\right)$. Maximum benefit cost ratio was recorded with pre emergence application of pendimethalin 30\% EC @ $4160 \mathrm{ml} \mathrm{ha}^{-1}$ (1250 g a.i.) followed by Propaquizafop 10\% EC @ $625 \mathrm{ml} \mathrm{ml}$ (62.5 g a.i.) ha ${ }^{-1}(2.60)$.

\section{Introduction}

There are several constraints in chilli production. Weed infestation is one of the major factors for loss in yield under irrigated conditions as chilli is usually planted at wider spacing and it grows very slowly during early growth stages. Weeds which emerge and become established during early stages of chilli growth can be very competitive and reduce the chilli yield potential significantly. Weeds are one of the major limiting factors in the production of chilli and one of the important spices as well as vegetable crops of 
the country grown over an area of 37,958 ha with a production of 5.54 lakh tonnes with a productivity of $14.59 \mathrm{t} \mathrm{ha}^{-1}$ in Karnataka (Anon., 2012). But, the extent of yield reduction caused by weeds vary from 30 to 80 $\%$ depending on type of weeds, season, variety, soil type, rainfall, duration and time of weed competition.

Mechanical methods of weed control are become laborious; time consuming and expensive on account of scarcity, of labour and the use of herbicides appears to be better substitute for this age old practice and a viable and effective method of weed control. The prolonged and indiscriminate use of recommended herbicides is observed to result in increased accumulation of their residues in soil with negative impact on crop growth factors and resulted in weed shift in some areas.

Thus, there is need to have alternate herbicides which may provide wide range of weed management to avoid weed shift also possible development of herbicide -resistant weeds. Propaquizapop is one of the most promising post emergence herbicides for effective weed management in chilli against complex graminaceous weed flora. Thus, keeping the above facts in view, the present investigation was carried out to evaluate the post emergence application of propaquizapop coupled with other herbicide and intercultivation for weed management in chilli.

\section{Materials and Methods}

The field experiment was conducted at Main Agricultural Research Station Farm, University of Agricultural Sciences, Raichur in Hyderabad Karnataka region in chilli. The soil was clayey with alkaline $\mathrm{pH}$ (8.00) having $0.75 \%$ OC, $269.0 \mathrm{~kg} \mathrm{ha}^{-1}$ available $\mathrm{N}$, $17.75 \mathrm{~kg} \mathrm{ha}^{-1}$ available $\mathrm{P}_{2} \mathrm{O}_{5}$ and $458.40 \mathrm{~kg}$ $\mathrm{ha}^{-1} \mathrm{~K}_{2} \mathrm{O}$. The study was conducted to know the bio-efficacy and selectivity of different herbicides for weed control in chilli. An experiment comprising ten treatments viz. Propaquizafop 10\% EC PoE (postemergence) @ $500 \mathrm{ml}$ per ha $\left(\mathrm{T}_{1}: 50 \mathrm{~g}\right.$ a.i. $)$, Propaquizafop 10\% EC PoE @ $625 \mathrm{ml} \mathrm{ha}^{-1}$ ( $\mathrm{T}_{2}: 62.5 \mathrm{~g}$ a.i.), Propaquizafop 10\% EC PoE @ $750 \mathrm{ml} \mathrm{ha}^{-1}\left(\mathrm{~T}_{3}: 75 \mathrm{~g}\right.$ a.i.), Propaquizafop 10\% EC PoE @ $1250 \mathrm{ml} \mathrm{ha}^{-1}$ ( $\mathrm{T}_{4}: 125 \mathrm{~g}$ a.i.), Pendimethalin 30\% EC PRE (preemergence) @ $4160 \mathrm{ml} \mathrm{ha}{ }^{-1} \quad\left(\mathrm{~T}_{5}: 1250 \mathrm{~g}\right.$ a.i. $)$, Pendimethalin 30\% EC PRE @ $4160 \mathrm{ml} \mathrm{ha}^{-1}$ $\left(1250 \mathrm{~g}\right.$ a.i.) $-1 \mathrm{IC}$ at 30 DAT $\left(\mathrm{T}_{6}\right)$, Propaquizafop 10\% EC PoE @ $625 \mathrm{ml} \mathrm{ha}^{-1}$ $(62.5 \mathrm{~g}$ a.i.) $\mathrm{fb}$ (followed by) 1 intercultivation (IC) at 30 DAT $\left(\mathrm{T}_{7}\right)$, Pendimethalin 30\% EC PRE @ $4160 \mathrm{ml} \mathrm{ha}^{-1}$ (1250 g a.i.) fb $\mathrm{T}_{2}$ at 2-7 leaf stage $\left(\mathrm{T}_{8}\right)$, weedy check $\left(\mathrm{T}_{9}\right)$ and control $\left(\mathrm{T}_{10}\right.$ : Hand weeding) laid out in Randomized complete block design (RCBD) with three replications.

Chilli crop was planted at a spacing of $75 \mathrm{~cm}$ $\mathrm{X} 45 \mathrm{~cm}$ and the recommended package of practices was performed during the crop period as per need. Pre emergent (PRE) application of pendimethalin $30 \%$ EC was carried out on the day of transplanting and post emergent (PoE) spray of propaquizafop $10 \%$ EC was applied at 2-7 leaf stage of weed (20 days after transplanting). Data on weed population species wise were recorded at pre treatment, 15, 30 and 45 days after spraying using quadrants of $0.5 \mathrm{~m} \times 0.5 \mathrm{~m}$, and likewise weed dry weight was recorded. Weed control efficiency (WCE) was worked out taking weed count into consideration. Further, data on weed density and dry weight was subjected to square root transformation before analysis.

The observation on phyto-toxicity of the chemical at higher dosages was also recorded separately. The field experiment was laid out in Randomized Block Design with prescribed 
treatments. The observation of phyto-toxicity on chilli and fodder cowpea plants were done on the basis of phytotoxicity rating scale (PRS) for the applied testing herbicide Propaquizafop $10 \%$ EC at 1, 3, 5, 7 and 10 DAA. The parameters on phytotoxicity were taken as vein clearing, leaf epinasty and hyponasty, necrosis (leaf tips and margins) and wilting. The observation on the level of phytotoxicity through visual assessment of crop response was rated in the scale of 0-10 (0 $=$ No adverse effect of herbicide on cotton and $10=100 \%$ adverse effect of herbicide on cotton).

The cost of production, gross returns, net returns and benefit cost ratio was worked out on the basis of actual production cost and yield of green chilli. All the parameters were subjected for statistical analysis and interpretation as outlined by (Panse and Sukhatme, 1967).

\section{Results and Discussion}

Weed flora: The prominent weed flora observed during the investigation were Dinebra retroflexa, Panicum flavidum, Panicum javanicum, Brachiaria reptans, Cynondon dactylon among monocots; Cyperus rotundus among sedge and Trianthema portulacastrum, Digeria aravensis, Amaranthus polygamus, Amaranthus viridis, Parthenium hysterophorus, Euphorbia hirta, Phyllannthus maderaspatensis, Portulaca oleracea, Sida acuta and Celosia argenstia among dicot weeds. These results are in line with Gare et al., (2015).

Effect on weeds: All the weed control treatments resulted significant reduction in weed growth in terms of density and dry weight as compared to weedy check (Table 1 $\& 2$ ). Among the different treatments, hand weeding thrice at 35, 50 and 65 days after transplanting (DAT) recorded lowest density and dry weight of weeds $\left(12.52 \mathrm{~m}^{-2}\right.$ and 19.99 g $\mathrm{m}^{-2}$, respectively) followed by pendimethalin 30\% EC PRE @ $4160 \mathrm{ml} \mathrm{ha}^{-1}$ (1250 g a.i.) fb one IC at 30 DAT $\left(18.45 \mathrm{~m}^{-2}\right)$ w.r.t. to density and pendimethalin 30\% EC PRE @ $4160 \mathrm{ml} \mathrm{ha}{ }^{-1}(1250 \mathrm{~g}$ a.i.) fb propaquizafop 10\% EC PoE @ $625 \mathrm{ml} \mathrm{ha}^{-1}$ $\left(24.20 \mathrm{~g} \mathrm{~m}^{-2}\right)$ with respect to dry weight.

Among the herbicidal treatments, the latter treatment i.e. pendimethalin 30\% EC PRE @ $4160 \mathrm{ml} \mathrm{ha}^{-1}$ (1250 $\mathrm{g}$ a.i.) fb one IC at 30 DAT recorded least number of weeds and it was at par with pendimethalin 30\% EC PRE @ $4160 \mathrm{ml} \mathrm{ha}^{-1}$ (1250 g a.i.) fb propaquizafop 10\% EC PoE@625 ml ha ${ }^{-1}$ (62.5 g a.i.) resulted in effective control of composite weed flora, thereby reduced the dry weight of weeds at 45 days after spraying (Table $1 \& 2$ ). These results are also in accordance with those of Hassan and Ahmad (2005) and Abid Khan et al., (2012) who also found highest number of weeds and dry weight of weeds $\mathrm{m}^{-2}$ in weedy check plots and lowest in hand weeded treatments.

Hand weeding thrice at 35, 50 and 65 DAT recorded higher weed control efficiency (WCE) at 45 days after spraying (88.17\%) followed by the treatment pendimethalin 30\% EC PRE @ $4160 \mathrm{ml} \mathrm{ha}^{-1}$ (1250 g a.i.) fb one IC at 30 DAT $(82.53 \%)$ and it was on par with pendimethalin 30\% EC PRE @ $4160 \mathrm{ml}$ $\mathrm{ha}^{-1}$ (1250 g a.i.) fb propaquizafop 10\% EC @ $625 \mathrm{ml} \mathrm{ha}^{-1}$ (62.5 g a.i.) over other treatments (Table 3). The results are analogous to those reported by Mekki et al., (2010), Arvadiya et al., (2012) and Gare et al., (2015).

Phytotoxicity on chilli and succeeding crop: The application of pendimethalin 30\% EC PRE @ $4160 \mathrm{ml} \mathrm{ha}{ }^{-1}(1250$ g a.i.) propaquizafop 10\% EC PoE @ $625 \mathrm{ml}$ ha and propaquizafop 10\% EC PoE @ 1250 ml $\mathrm{ha}^{-1}$ has not recorded any phyto-toxicity on 
chilli crop. After the harvest of chilli, fodder cowpea was grown to evaluate the herbicide carry over effect. Observations on germination, plant height and phyto-toxicity was recorded on succeeding fodder cowpea in those plots where different treatments of Pendimethalin 30\% EC and Propaquizafop $10 \%$ EC was applied on Chilli crop in the previous season and data indicates no adverse effect of Propaquizafop $10 \%$ EC treatment on cowpea as there was no significant difference on germination, plant height as well as no phyto-toxic symptoms like epinasty, hyponasty, necrosis, wilting and vein clearing were observed on cowpea crop.

The yield data also did not vary significantly among the treatments, where the testing herbicides were used in different doses 50 , 62.5, 75 and $125 \mathrm{~g}$ a.i. $\mathrm{ha}^{-1}$ in the previous chilli crop.

Effect weed control methods on growth and yield attributes and yield of chilli: In general, all weed control treatments had marked improvement on green chilli yield and the maximum green chilli yield $\left(23.2 \mathrm{t} \mathrm{ha}^{-1}\right)$ was recorded with hand weedings three at 35,50 and 65 DAT (Table 4) and it was on par with pendimethalin 30\% EC PRE @ $4160 \mathrm{ml} \mathrm{ha}^{-1}$ (1250 g a.i.) fb propaquizafop $10 \%$ EC PoE @ $625 \mathrm{ml} \mathrm{ha}^{-1}\left(22.5 \mathrm{t} \mathrm{ha}^{-1}\right)$ and pendimethalin 30\% EC PRE @ $4160 \mathrm{ml} \mathrm{ha}^{-1}$ (1250 g a.i.) fb one IC at 30 DAT $\left(20.8 \mathrm{t} \mathrm{ha}^{-1}\right)$ while the lowest green chilli yield was recorded with weedy check $\left(13.5 \mathrm{t} \mathrm{ha}^{-1}\right)$.

This beneficial effect of weed control measures in increasing green chilli yield could be attributed to suppression of weeds and inturn reduced soil moisture and nutrient losses due to weeds and making available to chiili crop. These finding are in agreement with the results of Deshpande et al., (2006), Kunti and Singh (2012) and Gare et al., (2015).

\section{Economics}

Economic evaluation of weed control measures indicated that thrice hand weeding at 35, 50 and 65 DAT recorded significantly higher gross returns (Rs. 1,97,200 ha ${ }^{-1}$ ) over weedy check (Rs. 1,15,033). Significantly highest net returns and benefit cost ratio (Rs. $1,17,470 \mathrm{ha}^{-1}$ and 2.60 , respectively) was recorded with pendimethalin 30\% EC PRE @ $4160 \mathrm{ml} \mathrm{ha}^{-1}$ (1250 g a.i.) fb propaquizafop $10 \%$ EC PoE @ $625 \mathrm{ml} \mathrm{ha}^{-1}$ and it was on par with that thrice hand weeding at 35,50 and 65 DAT (Rs. 1, 16,675 $\mathrm{ha}^{-1}$ and 2.45) and pendimethalin 30\% EC PRE @ $4160 \mathrm{ml} \mathrm{ha}^{-1}$ (1250 g a.i.) fb one IC at 30 DAT (Rs. $1,00,518 \mathrm{ha}^{-1}$ and 2.31, respectively).

These improved monitory returns were attributed to higher green chilli yield as a consequence of effective weed management methods. Similar results are obtained by Gare et al., (2015).

On the basis of experimentation, it was concluded that application of pendimethalin 30\% EC PRE @ $4160 \mathrm{ml} \mathrm{ha}^{-1}$ (1250 g a.i.) fb propaquizafop $10 \% \mathrm{EC} \mathrm{PoE}$ at 2-7 leaf stage of weeds @ $625 \mathrm{ml} \mathrm{ha}^{-1}$ (62.5 g a.i.) and pendimethalin 30\% EC PRE @ $4160 \mathrm{ml} \mathrm{ha}^{-1}$ (1250 $\mathrm{g}$ a.i.) fb one IC at 30 DAT in the labour scarce area instead of thrice hand weeding at at 35, 50 and 65 days after transplanting were more efficient, practically convenient and economically feasible method of weed control. 
Table.1 Effect of Propaquizafop 10\% EC and other treatments on density of weeds in chilli at different stages

\begin{tabular}{|c|c|c|c|c|c|c|c|c|c|c|c|c|c|c|c|c|}
\hline \multirow[t]{3}{*}{ Treatments } & \multicolumn{16}{|c|}{ Density of weeds (No. m $^{-2}$ ) } \\
\hline & \multicolumn{4}{|c|}{ Grasses } & \multicolumn{4}{|c|}{ Sedges } & \multicolumn{4}{|c|}{ Broad leaved } & \multicolumn{4}{|c|}{ Total } \\
\hline & BS & $\begin{array}{c}15 \\
\text { DAS }\end{array}$ & $\begin{array}{c}30 \\
\text { DAS }\end{array}$ & $\begin{array}{c}45 \\
\text { DAS }\end{array}$ & BS & $\begin{array}{c}15 \\
\text { DAS }\end{array}$ & $\begin{array}{c}30 \\
\text { DAS }\end{array}$ & $\begin{array}{c}45 \\
\text { DAS }\end{array}$ & BS & $\begin{array}{c}15 \\
\text { DAS }\end{array}$ & $\begin{array}{c}\text { 30 } \\
\text { DAS }\end{array}$ & $\begin{array}{c}45 \\
\text { DAS }\end{array}$ & BS & $\begin{array}{c}15 \\
\text { DAS }\end{array}$ & $\begin{array}{c}30 \\
\text { DAS }\end{array}$ & $\begin{array}{c}45 \\
\text { DAS }\end{array}$ \\
\hline $\mathrm{T}_{1}$ & $\begin{array}{c}6.47 \\
(41.67)\end{array}$ & $\begin{array}{c}5.47 \\
(29.67)\end{array}$ & $\begin{array}{c}4.39 \\
(19.00)\end{array}$ & $\begin{array}{c}3.67 \\
(13.18)\end{array}$ & $\begin{array}{c}1.94 \\
(3.50)\end{array}$ & $\begin{array}{c}1.66 \\
(2.50)\end{array}$ & $\begin{array}{c}1.55 \\
(2.17)\end{array}$ & $\begin{array}{c}1.66 \\
(2.50)\end{array}$ & $\begin{array}{c}6.16 \\
(37.67)\end{array}$ & $\begin{array}{c}6.37 \\
(40.33)\end{array}$ & $\begin{array}{c}6.53 \\
(42.33)\end{array}$ & $\begin{array}{c}6.42 \\
(41.00)\end{array}$ & $\begin{array}{c}9.12 \\
(82.83)\end{array}$ & $\begin{array}{c}8.53 \\
(72.50)\end{array}$ & $\begin{array}{c}7.98 \\
(63.50)\end{array}$ & $\begin{array}{c}7.55 \\
(56.68)\end{array}$ \\
\hline $\mathrm{T}_{2}$ & $\begin{array}{c}6.68 \\
(44.33)\end{array}$ & $\begin{array}{c}5.24 \\
(27.25)\end{array}$ & $\begin{array}{c}4.07 \\
(16.33)\end{array}$ & $\begin{array}{c}3.54 \\
(12.27)\end{array}$ & $\begin{array}{c}1.94 \\
(3.50)\end{array}$ & $\begin{array}{c}1.53 \\
(2.10)\end{array}$ & $\begin{array}{c}1.38 \\
(1.67)\end{array}$ & $\begin{array}{c}1.44 \\
(1.83)\end{array}$ & $\begin{array}{c}6.13 \\
(37.33)\end{array}$ & $\begin{array}{c}6.29 \\
(39.33)\end{array}$ & $\begin{array}{c}6.37 \\
(40.33)\end{array}$ & $\begin{array}{c}6.37 \\
(40.33)\end{array}$ & $\begin{array}{c}9.24 \\
(85.17)\end{array}$ & $\begin{array}{c}8.30 \\
(68.68)\end{array}$ & $\begin{array}{c}7.65 \\
(58.33)\end{array}$ & $\begin{array}{c}7.39 \\
(54.43)\end{array}$ \\
\hline $\mathrm{T}_{3}$ & $\begin{array}{c}6.79 \\
(45.80)\end{array}$ & $\begin{array}{c}5.19 \\
(26.67)\end{array}$ & $\begin{array}{c}3.85 \\
(14.58)\end{array}$ & $\begin{array}{c}3.25 \\
(10.30)\end{array}$ & $\begin{array}{c}1.85 \\
(3.17)\end{array}$ & $\begin{array}{c}1.48 \\
(1.93)\end{array}$ & $\begin{array}{c}1.32 \\
(1.50)\end{array}$ & $\begin{array}{c}1.41 \\
(1.75)\end{array}$ & $\begin{array}{c}6.26 \\
(39.00)\end{array}$ & $\begin{array}{c}6.26 \\
(39.00)\end{array}$ & $\begin{array}{c}6.40 \\
(40.67)\end{array}$ & $\begin{array}{c}6.40 \\
(40.67)\end{array}$ & $\begin{array}{c}9.39 \\
(87.97)\end{array}$ & $\begin{array}{c}8.24 \\
(67.60)\end{array}$ & $\begin{array}{c}7.55 \\
(56.75)\end{array}$ & $\begin{array}{c}7.28 \\
(52.72)\end{array}$ \\
\hline $\mathrm{T}_{4}$ & $\begin{array}{c}6.46 \\
(41.50)\end{array}$ & $\begin{array}{c}4.90 \\
(23.75)\end{array}$ & $\begin{array}{c}3.64 \\
(13.00)\end{array}$ & $\begin{array}{c}3.03 \\
(8.93)\end{array}$ & $\begin{array}{c}1.67 \\
(2.53)\end{array}$ & $\begin{array}{c}1.39 \\
(1.68)\end{array}$ & $\begin{array}{c}1.29 \\
(1.42)\end{array}$ & $\begin{array}{c}1.38 \\
(1.67)\end{array}$ & $\begin{array}{c}6.16 \\
(37.67)\end{array}$ & $\begin{array}{c}6.29 \\
(39.33)\end{array}$ & $\begin{array}{c}6.45 \\
(41.33)\end{array}$ & $\begin{array}{c}6.32 \\
(39.67)\end{array}$ & $\begin{array}{c}9.05 \\
(81.70)\end{array}$ & $\begin{array}{c}8.06 \\
(64.77)\end{array}$ & $\begin{array}{c}7.48 \\
(55.75)\end{array}$ & $\begin{array}{c}7.11 \\
(50.27)\end{array}$ \\
\hline $\mathrm{T}_{5}$ & $\begin{array}{c}4.24 \\
(17.73)\end{array}$ & $\begin{array}{c}3.72 \\
(13.62)\end{array}$ & $\begin{array}{c}3.35 \\
(10.97)\end{array}$ & $\begin{array}{c}3.32 \\
(10.77)\end{array}$ & $\begin{array}{c}1.64 \\
(2.43)\end{array}$ & $\begin{array}{c}1.44 \\
(1.83)\end{array}$ & $\begin{array}{c}1.36 \\
(1.60)\end{array}$ & $\begin{array}{c}1.35 \\
(1.58)\end{array}$ & $\begin{array}{c}3.35 \\
(11.00)\end{array}$ & $\begin{array}{c}3.30 \\
(10.67)\end{array}$ & $\begin{array}{c}3.15 \\
(9.67)\end{array}$ & $\begin{array}{c}3.20 \\
(10.00)\end{array}$ & $\begin{array}{c}5.61 \\
(31.17)\end{array}$ & $\begin{array}{c}5.13 \\
(26.12)\end{array}$ & $\begin{array}{c}4.74 \\
(22.23)\end{array}$ & $\begin{array}{c}4.75 \\
(22.35)\end{array}$ \\
\hline $\mathrm{T}_{6}$ & $\begin{array}{c}4.31 \\
(18.30)\end{array}$ & $\begin{array}{c}3.46 \\
(11.73)\end{array}$ & $\begin{array}{c}2.97 \\
(8.57)\end{array}$ & $\begin{array}{c}2.74 \\
(7.28)\end{array}$ & $\begin{array}{c}1.47 \\
(1.90)\end{array}$ & $\begin{array}{c}1.28 \\
(1.38)\end{array}$ & $\begin{array}{c}1.22 \\
(1.23)\end{array}$ & $\begin{array}{c}1.19 \\
(1.17)\end{array}$ & $\begin{array}{c}3.50 \\
(12.00)\end{array}$ & $\begin{array}{c}3.25 \\
(10.33)\end{array}$ & $\begin{array}{c}2.99 \\
(8.67)\end{array}$ & $\begin{array}{c}3.20 \\
(10.00)\end{array}$ & $\begin{array}{c}5.71 \\
(32.30)\end{array}$ & $\begin{array}{c}4.87 \\
(23.45)\end{array}$ & $\begin{array}{c}4.33 \\
(18.47)\end{array}$ & $\begin{array}{c}4.32 \\
(18.45)\end{array}$ \\
\hline $\mathrm{T}_{7}$ & $\begin{array}{c}6.35 \\
(40.13)\end{array}$ & $\begin{array}{c}3.93 \\
(15.17)\end{array}$ & $\begin{array}{c}3.10 \\
(9.33)\end{array}$ & $\begin{array}{c}2.89 \\
(8.08)\end{array}$ & $\begin{array}{c}1.68 \\
(2.57)\end{array}$ & $\begin{array}{c}1.38 \\
(1.67)\end{array}$ & $\begin{array}{c}1.37 \\
(1.63)\end{array}$ & $\begin{array}{c}1.24 \\
(1.30)\end{array}$ & $\begin{array}{c}5.12 \\
(26.00)\end{array}$ & $\begin{array}{c}4.03 \\
(16.00)\end{array}$ & $\begin{array}{c}3.82 \\
(14.33)\end{array}$ & $\begin{array}{c}3.82 \\
(14.33)\end{array}$ & $\begin{array}{c}8.29 \\
(68.47)\end{array}$ & $\begin{array}{c}5.75 \\
(32.83)\end{array}$ & $\begin{array}{c}5.05 \\
(25.30)\end{array}$ & $\begin{array}{c}4.90 \\
(23.72)\end{array}$ \\
\hline $\mathrm{T}_{8}$ & $\begin{array}{c}4.36 \\
(18.80)\end{array}$ & $\begin{array}{c}3.38 \\
(11.17)\end{array}$ & $\begin{array}{c}2.81 \\
(7.65)\end{array}$ & $\begin{array}{c}2.60 \\
(6.53)\end{array}$ & $\begin{array}{c}1.47 \\
(1.90)\end{array}$ & $\begin{array}{c}1.37 \\
(1.63)\end{array}$ & $\begin{array}{c}1.31 \\
(1.47)\end{array}$ & $\begin{array}{c}1.20 \\
(1.20)\end{array}$ & $\begin{array}{c}3.35 \\
(11.00)\end{array}$ & $\begin{array}{c}3.45 \\
(11.67)\end{array}$ & $\begin{array}{c}2.87 \\
(8.00)\end{array}$ & $\begin{array}{c}3.50 \\
(12.00)\end{array}$ & $\begin{array}{c}5.65 \\
(31.70)\end{array}$ & $\begin{array}{c}4.97 \\
(24.47)\end{array}$ & $\begin{array}{c}4.17 \\
(17.12)\end{array}$ & $\begin{array}{c}4.47 \\
(19.73)\end{array}$ \\
\hline $\mathrm{T}_{9}$ & $\begin{array}{c}6.80 \\
(46.00)\end{array}$ & $\begin{array}{c}7.42 \\
(54.85)\end{array}$ & $\begin{array}{c}7.75 \\
(59.83)\end{array}$ & $\begin{array}{c}7.62 \\
(57.83)\end{array}$ & $\begin{array}{c}1.61 \\
(2.33)\end{array}$ & $\begin{array}{c}1.85 \\
(3.17)\end{array}$ & $\begin{array}{c}1.89 \\
(3.33)\end{array}$ & $\begin{array}{c}1.94 \\
(3.50)\end{array}$ & $\begin{array}{c}6.21 \\
(38.33)\end{array}$ & $\begin{array}{c}6.45 \\
(41.33)\end{array}$ & $\begin{array}{c}6.63 \\
(43.67)\end{array}$ & $\begin{array}{c}6.68 \\
(44.33)\end{array}$ & $\begin{array}{c}9.32 \\
(86.67)\end{array}$ & $\begin{array}{c}9.98 \\
(99.35)\end{array}$ & $\begin{array}{c}10.35 \\
(106.83)\end{array}$ & $\begin{array}{c}10.29 \\
(105.67)\end{array}$ \\
\hline $\mathrm{T}_{10}$ & $\begin{array}{c}4.16 \\
(17.03)\end{array}$ & $\begin{array}{c}2.97 \\
(8.58)\end{array}$ & $\begin{array}{c}2.18 \\
(4.48)\end{array}$ & $\begin{array}{c}2.09 \\
(4.10)\end{array}$ & $\begin{array}{c}1.45 \\
(1.87)\end{array}$ & $\begin{array}{c}1.32 \\
(1.50)\end{array}$ & $\begin{array}{c}1.05 \\
(0.85)\end{array}$ & $\begin{array}{c}1.15 \\
(1.08)\end{array}$ & $\begin{array}{c}3.55 \\
(12.33)\end{array}$ & $\begin{array}{c}3.10 \\
(9.33)\end{array}$ & $\begin{array}{c}2.57 \\
(6.33)\end{array}$ & $\begin{array}{c}2.75 \\
(7.33)\end{array}$ & $\begin{array}{c}5.61 \\
(31.23)\end{array}$ & $\begin{array}{c}4.43 \\
(19.42)\end{array}$ & $\begin{array}{c}3.45 \\
(11.67)\end{array}$ & $\begin{array}{c}3.57 \\
(12.52)\end{array}$ \\
\hline S.Em \pm & 0.09 & 0.11 & 0.10 & 0.11 & 0.05 & 0.08 & 0.11 & 0.09 & 0.22 & 0.16 & 0.14 & 0.12 & 0.17 & 0.15 & 0.14 & 0.10 \\
\hline C.D@ 5\% & 0.28 & 0.32 & 0.30 & 0.33 & 0.16 & 0.23 & 0.32 & 0.27 & 0.66 & 0.46 & 0.43 & 0.36 & 0.50 & 0.44 & 0.41 & 0.30 \\
\hline CV $(\%)$ & 2.90 & 4.04 & 4.65 & 5.61 & 5.60 & 8.94 & 13.42 & 10.90 & 7.69 & 5.54 & 5.26 & 4.37 & 3.81 & 3.79 & 3.85 & 2.83 \\
\hline
\end{tabular}

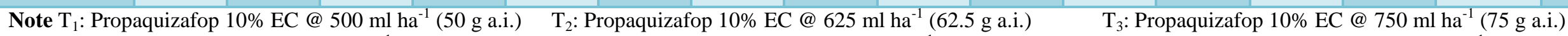

$\mathrm{T}_{4}$ : Propaquizafop 10\% EC @ $1250 \mathrm{ml} \mathrm{ha}^{-1}$ (125 g a.i.) $\quad \mathrm{T}_{5}$ : Pendimethalin 30\% EC @ $4160 \mathrm{ml} \mathrm{ha}^{-1}$ (1250 g a.i.)

$\mathrm{T}_{6}$ : Pendimethalin 30\% EC @ $4160 \mathrm{ml} \mathrm{ha}^{-1}$ (1250 g a.i.) $-1 \mathrm{IC}$ at 30 DAT

$\mathrm{T}_{7}$ : Propaquizafop 10\% EC @ $625 \mathrm{ml} \mathrm{ha}^{-1}(62.5 \mathrm{~g}$ a.i. $) \quad \mathrm{T}_{8}$ : Pendimethalin $30 \% \mathrm{EC} @ 4160 \mathrm{ml} \mathrm{ha}^{-1}(1250 \mathrm{~g}$ a.i. $)$ - 1 IC at 30 DAT 
Table.2 Effect of Propaquizafop 10\% EC and other treatments on weed dry weight at different growth stages in chilli

\begin{tabular}{|c|c|c|c|c|c|c|c|c|}
\hline \multirow[t]{3}{*}{ Treatments } & \multicolumn{8}{|c|}{ Weed dry weight $\left(\mathrm{g} \mathrm{m}^{-2}\right)$} \\
\hline & \multicolumn{4}{|c|}{ At 30 days after spraying } & \multicolumn{4}{|c|}{ At 45 days after spraying } \\
\hline & Grasses & Sedges & BLW & Total & Grasses & Sedges & BLW & Total \\
\hline $\mathrm{T}_{1}$ : Propaquizafop 10\% EC @ $500 \mathrm{ml} \mathrm{ha}^{-1}$ (50 g a.i.) & $\begin{array}{c}5.36 \\
(28.45)\end{array}$ & $\begin{array}{c}1.85 \\
(3.17)\end{array}$ & $\begin{array}{c}7.62 \\
(57.88)\end{array}$ & $\begin{array}{c}9.47 \\
(89.50)\end{array}$ & $\begin{array}{c}5.45 \\
(29.40)\end{array}$ & $\begin{array}{c}1.81 \\
(3.01)\end{array}$ & $\begin{array}{c}8.02 \\
(64.00)\end{array}$ & $\begin{array}{c}9.83 \\
(96.41)\end{array}$ \\
\hline $\mathrm{T}_{2}$ : Propaquizafop 10\% EC @ $625 \mathrm{ml} \mathrm{ha}^{-1}$ (62.5 g a.i.) & $\begin{array}{c}4.99 \\
(24.67)\end{array}$ & $\begin{array}{c}1.66 \\
(2.52)\end{array}$ & $\begin{array}{c}7.58 \\
(57.25)\end{array}$ & $\begin{array}{c}9.20 \\
(84.44)\end{array}$ & $\begin{array}{c}5.02 \\
(24.92)\end{array}$ & $\begin{array}{c}1.64 \\
(2.43)\end{array}$ & $\begin{array}{c}7.90 \\
(62.12)\end{array}$ & $\begin{array}{c}9.47 \\
(89.47)\end{array}$ \\
\hline $\mathrm{T}_{3}$ : Propaquizafop 10\% EC @ $750 \mathrm{ml} \mathrm{ha}{ }^{-1}$ (75 g a.i.) & $\begin{array}{c}4.69 \\
(21.72)\end{array}$ & $\begin{array}{c}1.56 \\
(2.17)\end{array}$ & $\begin{array}{c}7.54 \\
(56.67)\end{array}$ & $\begin{array}{c}8.99 \\
(80.56)\end{array}$ & $\begin{array}{c}4.80 \\
(22.78)\end{array}$ & $\begin{array}{c}1.61 \\
(2.35)\end{array}$ & $\begin{array}{c}8.00 \\
(63.72)\end{array}$ & $\begin{array}{c}9.44 \\
(88.86)\end{array}$ \\
\hline $\mathrm{T}_{4}$ : Propaquizafop 10\% EC @ $1250 \mathrm{ml} \mathrm{ha}^{-1}$ (125 g a.i.) & $\begin{array}{c}4.62 \\
(21.09)\end{array}$ & $\begin{array}{c}1.54 \\
(2.12)\end{array}$ & $\begin{array}{c}7.55 \\
(56.75)\end{array}$ & $\begin{array}{c}8.96 \\
(79.96)\end{array}$ & $\begin{array}{c}4.21 \\
(17.47)\end{array}$ & $\begin{array}{c}1.51 \\
(2.02)\end{array}$ & $\begin{array}{c}7.84 \\
(61.18)\end{array}$ & $\begin{array}{c}9.00 \\
(80.67)\end{array}$ \\
\hline $\mathrm{T}_{5}$ : Pendimethalin 30\% EC @ $4160 \mathrm{ml} \mathrm{ha}^{-1}$ (1250 g a.i.) & $\begin{array}{c}4.13 \\
(16.81)\end{array}$ & $\begin{array}{c}1.59 \\
(2.27)\end{array}$ & $\begin{array}{c}3.71 \\
(13.50)\end{array}$ & $\begin{array}{c}5.73 \\
(32.58)\end{array}$ & $\begin{array}{c}4.52 \\
(20.19)\end{array}$ & $\begin{array}{c}1.49 \\
(1.98)\end{array}$ & $\begin{array}{c}4.03 \\
(15.97)\end{array}$ & $\begin{array}{c}6.20 \\
(38.14)\end{array}$ \\
\hline $\begin{array}{l}\mathrm{T}_{6} \text { : Pendimethalin } 30 \% \mathrm{EC} @ 4160 \mathrm{ml} \mathrm{ha}^{-1}(1250 \mathrm{~g} \text { a.i. }) \\
\quad-1 \mathrm{IC} \text { at } 30 \mathrm{DAT}\end{array}$ & $\begin{array}{c}3.32 \\
(10.76)\end{array}$ & $\begin{array}{c}1.37 \\
(1.62)\end{array}$ & $\begin{array}{c}3.48 \\
(11.87)\end{array}$ & $\begin{array}{c}4.95 \\
(24.26)\end{array}$ & $\begin{array}{c}3.66 \\
(13.15)\end{array}$ & $\begin{array}{c}1.36 \\
(1.61)\end{array}$ & $\begin{array}{c}3.85 \\
(14.61)\end{array}$ & $\begin{array}{c}5.44 \\
(29.37)\end{array}$ \\
\hline $\begin{array}{l}\mathrm{T}_{7} \text { : Propaquizafop } 10 \% \text { EC @ } 625 \mathrm{ml} \mathrm{ha}^{-1}(62.5 \mathrm{~g} \text { a.i. }) \\
\quad \text { - } 1 \text { IC at } 30 \text { DAT }\end{array}$ & $\begin{array}{c}3.67 \\
(13.25)\end{array}$ & $\begin{array}{c}1.49 \\
(1.98)\end{array}$ & $\begin{array}{c}4.41 \\
(19.23)\end{array}$ & $\begin{array}{c}5.89 \\
(34.46)\end{array}$ & $\begin{array}{c}3.90 \\
(14.95)\end{array}$ & $\begin{array}{c}1.40 \\
(1.70)\end{array}$ & $\begin{array}{c}4.66 \\
(21.43)\end{array}$ & $\begin{array}{c}6.19 \\
(38.08)\end{array}$ \\
\hline $\begin{array}{l}\mathrm{T}_{8}: \text { Pendimethalin } 30 \% \mathrm{EC} @ 4160 \mathrm{ml} \mathrm{ha}^{-1}(1250 \mathrm{~g} \text { a.i. })- \\
\mathrm{T}_{2} \text { at } 2-7 \text { leaf stage }\end{array}$ & $\begin{array}{c}3.16 \\
(9.76)\end{array}$ & $\begin{array}{c}1.33 \\
(1.53)\end{array}$ & $\begin{array}{c}3.84 \\
(14.50)\end{array}$ & $\begin{array}{c}5.10 \\
(25.79)\end{array}$ & $\begin{array}{c}3.10 \\
(9.33)\end{array}$ & $\begin{array}{c}1.34 \\
(1.53)\end{array}$ & $\begin{array}{c}3.69 \\
(13.33)\end{array}$ & $\begin{array}{c}4.94 \\
(24.20)\end{array}$ \\
\hline $\mathrm{T}_{9}:$ Weedy check & $\begin{array}{c}7.64 \\
(58.10) \\
\end{array}$ & $\begin{array}{c}2.06 \\
(3.99)\end{array}$ & $\begin{array}{c}7.76 \\
(60.00)\end{array}$ & $\begin{array}{c}11.06 \\
(122.09)\end{array}$ & $\begin{array}{c}7.96 \\
(63.08) \\
\end{array}$ & $\begin{array}{c}2.18 \\
(4.52)\end{array}$ & $\begin{array}{c}8.07 \\
(64.92) \\
\end{array}$ & $\begin{array}{c}11.52 \\
(132.51)\end{array}$ \\
\hline $\mathrm{T}_{10}:$ Control (Hand weeding) & $\begin{array}{c}2.93 \\
(8.32)\end{array}$ & $\begin{array}{c}1.22 \\
(1.25)\end{array}$ & $\begin{array}{c}3.04 \\
(9.00)\end{array}$ & $\begin{array}{c}4.34 \\
(18.57)\end{array}$ & $\begin{array}{c}2.71 \\
(7.10)\end{array}$ & $\begin{array}{c}1.21 \\
(1.22)\end{array}$ & $\begin{array}{c}3.45 \\
(11.67)\end{array}$ & $\begin{array}{c}4.50 \\
(19.99)\end{array}$ \\
\hline S.Em \pm & 0.17 & 0.04 & 0.15 & 0.18 & 0.15 & 0.07 & 0.15 & 0.16 \\
\hline C.D@ 5\% & 0.50 & 0.13 & 0.45 & 0.53 & 0.44 & 0.21 & 0.44 & 0.47 \\
\hline $\mathrm{CV}(\%)$ & 6.62 & 4.88 & 4.59 & 4.16 & 5.61 & 8.03 & 4.28 & 3.61 \\
\hline
\end{tabular}

Note: DAT- Days after transplanting, IC- Intercultivation, DAS- Days after spraying, BS- Before spraying and figures in parenthesis indicates original values and outside are transformed values [square root of $(\mathrm{X}+1)$ ] 
Table.3 Effect of Propaquizafop 10\% EC and other treatments on weed control efficiency (\%) at different growth stages in chilli

\begin{tabular}{|c|c|c|c|c|c|c|c|c|}
\hline \multirow[t]{3}{*}{ Treatments } & \multicolumn{8}{|c|}{ Weed control efficiency $(\%)$} \\
\hline & \multicolumn{4}{|c|}{ At 30 days after spraying } & \multicolumn{4}{|c|}{ At 45 days after spraying } \\
\hline & Grasses & Sedges & BLW & Total & Grasses & Sedges & BLW & Total \\
\hline $\mathrm{T}_{1}$ : Propaquizafop 10\% EC @ $500 \mathrm{ml} \mathrm{ha}^{-1}$ (50 g a.i.) & 68.05 & 33.33 & 2.64 & 40.29 & 77.24 & 28.17 & 7.40 & 46.34 \\
\hline $\mathrm{T}_{2}$ : Propaquizafop 10\% EC @ $625 \mathrm{ml} \mathrm{ha}^{-1}$ (62.5 g a.i.) & 72.74 & 50.00 & 7.29 & 45.35 & 78.77 & 46.83 & 8.83 & 48.43 \\
\hline $\mathrm{T}_{3}$ : Propaquizafop 10\% EC @ $750 \mathrm{ml} \mathrm{ha}^{-1}$ (75 g a.i.) & 75.61 & 52.78 & 6.48 & 46.74 & 82.17 & 48.61 & 8.19 & 50.10 \\
\hline $\mathrm{T}_{4}$ : Propaquizafop 10\% EC @ $1250 \mathrm{ml} \mathrm{ha}^{-1}$ (125 g a.i.) & 78.26 & 55.56 & 4.37 & 47.47 & 84.56 & 51.79 & 10.60 & 52.45 \\
\hline $\mathrm{T}_{5}$ : Pendimethalin 30\% EC @ $4160 \mathrm{ml} \mathrm{ha}^{-1}$ (1250 g a.i.) & 81.62 & 50.83 & 77.83 & 79.13 & 81.36 & 54.17 & 77.43 & 78.85 \\
\hline $\begin{array}{l}\mathrm{T}_{6}: \text { Pendimethalin } 30 \% \text { EC @ } 4160 \mathrm{ml} \mathrm{ha}^{-1} \text { (1250 g a.i.) } \\
\quad-1 \text { IC at } 30 \text { DAT }\end{array}$ & 85.66 & 63.06 & 80.01 & 82.67 & 87.38 & 66.27 & 77.48 & 82.53 \\
\hline $\begin{array}{l}\mathrm{T}_{7} \text { : Propaquizafop 10\% EC @ } 625 \mathrm{ml} \mathrm{ha}^{-1}(62.5 \mathrm{~g} \text { a.i. }) \\
\text { - } 1 \text { IC at } 30 \text { DAT }\end{array}$ & 84.35 & 49.72 & 66.69 & 76.15 & 86.00 & 61.67 & 67.79 & 77.57 \\
\hline $\begin{array}{l}\mathrm{T}_{8}: \text { Pendimethalin } 30 \% \mathrm{EC} @ 4160 \mathrm{ml} \mathrm{ha}^{-1}(1250 \mathrm{~g} \text { a.i. })- \\
\mathrm{T}_{2} \text { at } 2-7 \text { leaf stage }\end{array}$ & 87.24 & 55.28 & 81.60 & 83.98 & 88.65 & 65.48 & 72.95 & 81.33 \\
\hline $\mathrm{T}_{9}$ : Weedy check & 0.00 & 0.00 & 0.00 & 0.00 & 0.00 & 0.00 & 0.00 & 0.00 \\
\hline \multirow[t]{2}{*}{$\mathrm{T}_{10}$ : Control (Hand weeding) } & 92.52 & 73.89 & 85.41 & 89.08 & 92.93 & 67.56 & 83.51 & 88.17 \\
\hline & 1.32 & 5.91 & 3.82 & 1.83 & 1.10 & 4.93 & 2.81 & 1.14 \\
\hline C.D@ 5\% & 3.91 & 17.55 & 11.34 & 5.43 & 3.28 & 14.64 & 8.35 & 3.40 \\
\hline CV (\%) & 3.14 & 21.11 & 16.04 & 5.35 & 2.52 & 17.39 & 11.75 & 3.27 \\
\hline
\end{tabular}

Note: DAT- Days after transplanting, IC- Intercultivation, DAS- Days after spraying and BLWS- Broad leaved weeds 
Table.4 Ancillary characters, yield and economics of chilli as influenced by different weed control treatments

\begin{tabular}{|c|c|c|c|c|c|c|c|}
\hline \multirow[t]{3}{*}{ Treatments } & \multicolumn{3}{|c|}{ Ancillary characters and yield } & \multicolumn{4}{|c|}{ Economics } \\
\hline & \multirow[t]{2}{*}{$\begin{array}{c}\text { Plant } \\
\text { height }(\mathbf{c m})\end{array}$} & \multirow[t]{2}{*}{$\begin{array}{c}\text { No of } \\
\text { branches }\end{array}$} & \multirow[t]{2}{*}{$\begin{array}{l}\text { Yield } \\
(\mathbf{t} / \mathbf{h a})\end{array}$} & $\begin{array}{c}\text { Cost of } \\
\text { Cultivation }\end{array}$ & $\begin{array}{c}\text { Gross } \\
\text { returns }\end{array}$ & $\begin{array}{l}\text { Net } \\
\text { returns }\end{array}$ & \multirow[t]{2}{*}{$\mathbf{B}: \mathbf{C}$} \\
\hline & & & & \multicolumn{3}{|c|}{ Rs. ha ${ }^{-1}$} & \\
\hline $\mathrm{T}_{1}$ : Propaquizafop 10\% EC @ $500 \mathrm{ml} \mathrm{ha}^{-1}$ (50 g a.i.) & 81.8 & 16.3 & 17.5 & 70797 & 148750 & 77953 & 2.10 \\
\hline $\mathrm{T}_{2}$ : Propaquizafop 10\% EC @ $625 \mathrm{ml} \mathrm{ha}^{-1}$ (62.5 g a.i.) & 83.3 & 17.0 & 17.9 & 71020 & 152150 & 81130 & 2.15 \\
\hline $\mathrm{T}_{3}$ : Propaquizafop 10\% EC @ $750 \mathrm{ml} \mathrm{ha}^{-1}$ (75 g a.i.) & 84.2 & 17.2 & 18.2 & 71243 & 154417 & 83174 & 2.16 \\
\hline $\mathrm{T}_{4}$ : Propaquizafop 10\% EC @ $1250 \mathrm{ml} \mathrm{ha}^{-1}$ (125 g a.i.) & 84.8 & 17.4 & 18.5 & 72134 & 157533 & 85399 & 2.20 \\
\hline $\mathrm{T}_{5}$ : Pendimethalin 30\% EC @ $4160 \mathrm{ml} \mathrm{ha}^{-1}$ (1250 g a.i.) & 87.1 & 19.2 & 20.4 & 72133 & 173400 & 101267 & 2.40 \\
\hline $\begin{array}{l}\mathrm{T}_{6}: \text { Pendimethalin } 30 \% \mathrm{EC} @ 4160 \mathrm{ml} \mathrm{ha}^{-1}(1250 \mathrm{~g} \text { a.i. }) \\
-1 \mathrm{IC} \text { at } 30 \mathrm{DAT}\end{array}$ & 87.4 & 20.4 & 20.8 & 76565 & 177083 & 100518 & 2.31 \\
\hline $\begin{array}{l}\mathrm{T}_{7} \text { : Propaquizafop } 10 \% \text { EC @ } 625 \mathrm{ml} \mathrm{ha}^{-1}(62.5 \mathrm{~g} \text { a.i. }) \\
\quad-1 \text { IC at } 30 \text { DAT }\end{array}$ & 85.1 & 19.1 & 19.2 & 75674 & 163200 & 87526 & 2.16 \\
\hline $\begin{array}{l}\mathrm{T}_{8}: \text { Pendimethalin } 30 \% \mathrm{EC} @ 4160 \mathrm{ml} \mathrm{ha}^{-1}(1250 \mathrm{~g} \text { a.i. })- \\
\mathrm{T}_{2} \text { at } 2-7 \text { leaf stage }\end{array}$ & 87.5 & 20.6 & 22.5 & 73497 & 190967 & 117470 & 2.60 \\
\hline $\mathrm{T}_{9}:$ Weedy check & 69.0 & 15.8 & 13.5 & 64905 & 115033 & 50128 & 1.77 \\
\hline $\mathrm{T}_{10}:$ Control (Hand weeding) & 88.0 & 22.2 & 23.2 & 80525 & 197200 & 116675 & 2.45 \\
\hline S.Em \pm & 1.81 & 1.33 & 1.24 & 1247 & 10563 & 10364 & 0.14 \\
\hline C.D@ 5\% & 5.39 & 3.95 & 3.69 & 3706 & 31386 & 30793 & 0.42 \\
\hline CV (\%) & 3.75 & 12.44 & 11.23 & 2.97 & 11.23 & 19.92 & 11.10 \\
\hline
\end{tabular}

Note: DAT- Days after transplanting, IC- Intercultivation, DAS- Days after spraying, B:C- Benefit-cost ratio, Men and women labour @ Rs. 236 each, FYM @Rs. $600 \mathrm{~kg}^{-1}$, Urea @Rs. $5.72 \mathrm{~kg}^{-1}$, DAP @Rs. $25.32 \mathrm{~kg}^{-1}$, MoP @Rs. $18.22 \mathrm{~kg}^{-1}$, Pegasus $500 \mathrm{SC} @$ Rs. $3868 \mathrm{~kg}^{-1}$, Chloropyriphos @ Rs. $480 \mathrm{l}^{-1}, \mathrm{Spinosad}$ $@$ Rs.1250 per 75ml and Green chilli @ Rs $8.50 \mathrm{~kg}^{-1}$. 


\section{References}

Abid Khan, Muhammad Sajid, Zahid Hussain and Abdul Mateen Khattak, 2012, Effect of different weed control methods on weeds and yield of chillies (Capsicum annuum L.). Pakistan. J. Weed Sci. Res., 18(1): 71-78.

Anonymous, 2012, Horticulture Statistics of Karnataka State at a Glance 2011-12. http://www.horticulture.kar.nic.in.

Arvadiya, L. K., Raj, V. C., Patel, T. U. and Arvadia, M. K., 2012, Influence of plant population and weed management on weed flora and productivity of sweet corn. Indian $J$. Agron., 57(2): 162-267.

Deshpande, R. M., Pawar, W. S., Mankar, P. S., Bobade, P. N. and Chimote, A. N., 2006, Integrated weed management in rainfed cotton. Indian J. Agron., 51(1): 68-69.

Gare, B. N., Raundal, P. U. and Burli, A.V., 2015, Integrated weed management in rainfed chilli (Capsicum annum L.). Karnataka J. Agric. Sci., 28(2):164167.

Hassan, A.A.A. and M.K.A. Ahmed, 2005, The influence of some herbicides and additional hoeing in maize growth and yield and yield components. Int. J. Agric. Biol., 7(5): 708-711.

Kunti, G. S. and Singh, H. P., 2012, Weed management practices on growth and yield of winter season brinjal under Chhattisgarah plain conditions. Indian J. Weed Sci., 44(1): 18-20.

Mekki, B. B., Faida, A. A. and Kowthar, G., 2010, Effect of weed control treatments on yield and seed quality of some canola cultivars and associated weeds in newly reclaimed sandy soils. American-Eurasian J. Agric. \& Environ. Sci., 7(2): 202-209.

Panse, V. G. and Sukhatme, P. U., 1967, Statistical Methods for Agricultural Workers. ICAR, New Delhi.

\section{How to cite this article:}

Krishnamurthy. D, B.K. Desai and Maruthi. 2020. Integrated Weed Management in Chilli (Capsicum Annum L.). Int.J.Curr.Microbiol.App.Sci. 9(01): 2037-2045. doi: https://doi.org/10.20546/ijcmas.2020.901.231 